TU-862

\title{
Constraints on Scalar Phantoms
}

\author{
Masaki Asano and Ryuichiro Kitano \\ Department of Physics, Tohoku University, Sendai 980-8578, Japan
}

\begin{abstract}
We update the constraints on the minimal model of dark matter, where a stable real scalar field is added to the standard model Lagrangian with a renormalizable coupling to the Higgs field. Once we fix the dark matter abundance, there are only two relevant model parameters, the mass of the scalar field and that of the Higgs boson. The recent data from the CDMS II experiment have excluded a parameter region where the scalar field is light such as less than about $50 \mathrm{GeV}$. In a large parameter region, the consistency of the model can be tested by the combination of future direct detection experiments and the LHC experiments.
\end{abstract}


Weakly interacting massive particle (WIMP) is one of the most attractive scenarios to explain the dark matter component of the universe. The simplest and the most economical model to realize this scenario is the model of the scalar dark matter, where a new gauge singlet real scalar field $S$ is introduced to the standard model and it has an interaction with the Higgs field, $H$. The following Lagrangian density is added to the standard model:

$$
\mathcal{L}_{S}=\frac{1}{2} \partial_{\mu} S \partial^{\mu} S-\frac{m^{2}}{2} S^{2}-\frac{k}{2} S^{2}|H|^{2}-\frac{h}{4 !} S^{4} .
$$

This model has been first proposed in Ref. [1] (it is called scalar phantoms) and further studied in Refs. [2, 3, 6, 6, 6, 0, 8, 9]. One can obtain the correct size of the dark matter density via the standard thermal decoupling process in the early universe.

The model can be either thought of as a device to accommodate dark matter in new physics models or as a fundamental theory to describe the physics up to the Planck scale. If we take the latter attitude, the parameter region for $k$ and $h$ is subject to the constraint from perturbativity and stability of the potential [4, 5, 6, 9].

Because the model is compact, there are only four unknown parameters: the Higgs boson mass $m_{h}^{2}$, the mass of dark matter $m_{S}^{2}\left(\equiv m^{2}+k\langle H\rangle^{2}=m^{2}+k v^{2}\right)$, and two coupling constants $k$ and $h$. Here $v \simeq 174 \mathrm{GeV}$. Since the coupling constant of the dark matter self-interaction $h$ is not important for most of interesting physical observables, there are only three relevant parameters, one of which can be fixed by requiring the correct dark matter abundance. Therefore, the model is highly predictive. The parameter space is a two-dimensional $m_{h}-m_{S}$ plane.

In this article, we update the constraints on the model in light of new data from the CDMS II experiment [12] as well as the Tevatron exclusion [13] of a Higgs boson mass region. We discuss the interplay between the dark matter detection experiments and the Higgs boson searches at the LHC experiments.

The scalar field $S$ is stable due to a discrete symmetry $S \leftrightarrow-S$, and therefore is a candidate for a non-baryonic cold dark matter. In the WIMP scenario, the number density of $S$ is determined by the annihilation cross section, $\left\langle\sigma_{\text {ann. }} v\right\rangle$, which is proportional to $k^{2}$. One can fix the coupling constant $k$ by requiring the abundance to explain the dark matter component of the energy density of the universe [14]. The

\footnotetext{
*Similar studies have been done in Refs. [10, 11] very recently.
} 
energy density of dark matter is given by

$$
\Omega_{S} \simeq \frac{1.8 \times 10^{-10} \mathrm{GeV}^{-2}}{\left\langle\sigma_{\text {ann. }} v\right\rangle}
$$

The annihilation cross section is given by

$$
\left\langle\sigma_{\text {ann. }} v\right\rangle=\frac{4 k^{2} v^{2}}{\left(4 m_{S}^{2}-m_{h}^{2}\right)^{2}+m_{h}^{2} \Gamma_{H}^{2}} \cdot \frac{\left.\Gamma_{H}\right|_{m_{h}=2 m_{S}}}{2 m_{S}}
$$

for $m_{S}<m_{h}$, and

$$
\left\langle\sigma_{\text {ann. }} v\right\rangle=\frac{4 k^{2} v^{2}}{\left(4 m_{S}^{2}-m_{h}^{2}\right)^{2}+m_{h}^{2} \Gamma_{H}^{2}} \cdot \frac{\left.\Gamma_{H}\right|_{m_{h}=2 m_{S}}}{2 m_{S}}+\frac{k^{2} \sqrt{1-m_{h}^{2} / m_{S}^{2}}}{64 \pi m_{S}^{2}}\left(1+\frac{3 m_{h}^{2}}{4 m_{S}^{2}-m_{h}^{2}}\right),
$$

for $m_{S} \geq m_{h}$. Here $\Gamma_{H}$ and $\left.\Gamma_{H}\right|_{m_{h}=2 m_{S}}$ are the total decay width of the Higgs boson and that with a mass $2 m_{S}$, respectively. (One should take out $h \rightarrow S S$ mode in $\left.\left.\Gamma_{H}\right|_{m_{h}=2 m_{S}}.\right)$ By using the WMAP data [14], $\Omega_{S} \simeq 0.11$, one can fix $k$.

Dark matter $S$ interacts with nuclei through an exchange of the Higgs boson. Direct detection experiments such as CDMS II can therefore put constrains on the model. The spin-independent cross section of the $S$-nucleus elastic scattering normalized to a nucleon is given by

$$
\sigma_{S I}=\frac{1}{4 \pi} \frac{(1 \mathrm{GeV})^{2}}{A^{2} m_{S}^{2}}\left(Z f_{p}+(A-Z) f_{n}\right)^{2}
$$

where $A$ and $Z$ are mass and atomic numbers of the target nucleus, respectively. The $f_{p}$ and $f_{n}$ factors are given by

$$
f_{N}=\frac{k m_{N}}{m_{h}^{2}} \sum_{q} f_{q}^{N}, \quad \text { and } \quad f_{q}^{N}=\frac{m_{q}\langle N|\bar{q} q| N\rangle}{m_{N}}
$$

where $q=u, d, s, c, b, t$ and $N=p, n$. In this study, we use

$$
\begin{aligned}
& f_{u}^{p}=0.021, \quad f_{d}^{p}=0.029, \quad f_{s}^{p}=0.0 \\
& f_{u}^{n}=0.016, \quad f_{d}^{n}=0.037, \quad f_{s}^{n}=0.0 \\
& f_{c}^{p}=f_{b}^{p}=f_{t}^{p}=f_{c}^{n}=f_{b}^{n}=f_{t}^{n}=0.070
\end{aligned}
$$

which give a conservative estimate of the scattering cross section [15, 16, 17, 18].

The Higgs boson can decay into a pair of $S$ if $m_{S}<m_{h} / 2$. This invisible decay width is given by:

$$
\Gamma_{h \rightarrow S S}=\frac{k^{2} v^{2}}{16 \pi m_{h}} \sqrt{1-\frac{4 m_{S}^{2}}{m_{h}^{2}}}
$$




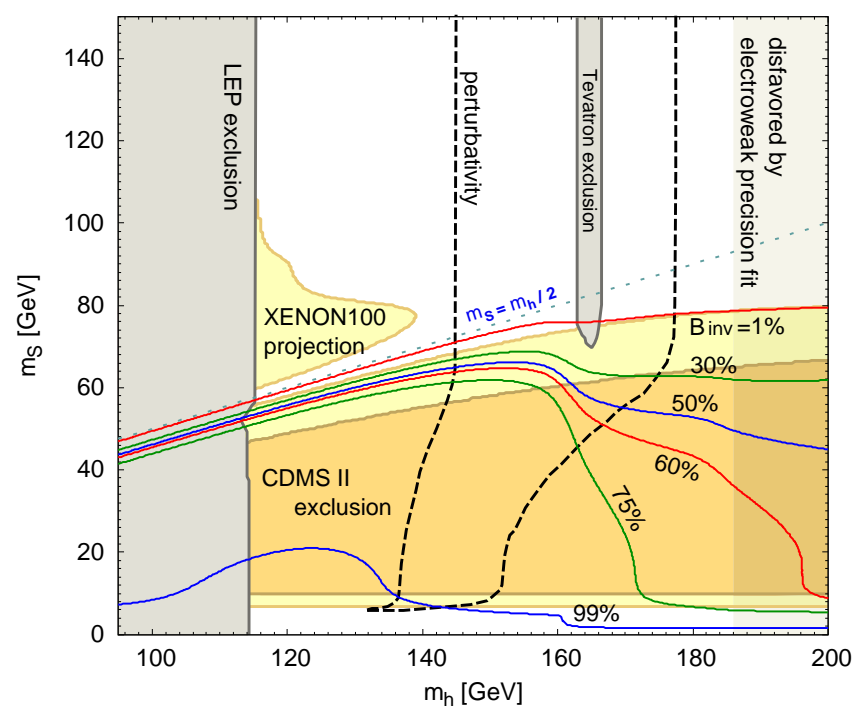

Figure 1: Experimental constraints and contours of the branching ratio of the invisible Higgs boson decay. The region inside the dashed lines satisfies the stability and triviality bounds for $h\left(m_{Z}\right)=0$.

The strategy to search for the Higgs boson in collider experiments will be tightly connected to dark matter physics in this model.

There have been studies of the invisible Higgs decay at the LHC. The discovery potential is a function of

$$
\xi^{2}=\frac{\sigma_{\mathrm{BSM}}}{\sigma_{\mathrm{SM}}} \cdot B_{\mathrm{inv}}
$$

where $B_{\mathrm{inv}}$ is the branching fraction of the invisible decay mode, and $\sigma_{\mathrm{BSM}}$ and $\sigma_{\mathrm{SM}}$ are the production cross sections of the Higgs boson in new physics models and in the standard model, respectively. At the ATLAS detector with $30 \mathrm{fb}^{-1}$, it is possible to discover the invisible decay of the Higgs boson in the $100 \mathrm{GeV}<m_{h}<200 \mathrm{GeV}$ region if $\xi^{2} \gtrsim 60 \%$ [19. In the present model, $\xi^{2}=B_{\text {inv }}$ because the production cross section is same as the one in the standard model.

Let us discuss the current experimental constraints on this model and the size of $B_{\text {inv }}$ which will be important for the Higgs boson search. As explained before, one can discuss the constraints/prediction of the model on the $m_{S}-m_{h}$ plane by requiring the correct dark matter abundance (Fig. 1) I. The recent data from the

\footnotetext{
${ }^{\dagger}$ In calculating the dark matter abundance and the decay width of the Higgs boson, we have used the program HDECAY [20].
} 
CDMS II experiment excludes the region with $10 \mathrm{GeV} \lesssim m_{S} \lesssim 50 \mathrm{GeV}$ although there are uncertainties from the local density of dark matter. We also overlaid the projected sensitivity of the XENON100 experiment [21]. The bound from the Higgs boson searches are also shown. The shaded region in the left side is excluded by the LEP-II direct Higgs boson searches [22, 23]. The excluded region from the Tevatron experiments, $163 \mathrm{GeV}<m_{h}<166 \mathrm{GeV}$ [13], is also shown where the bound disappears for $m_{S} \lesssim 70 \mathrm{GeV}$ due to large $B_{\text {inv }}$. The region $m_{h}>186 \mathrm{GeV}$ is disfavored by the electroweak precision measurements (95\% CL) [24]. A very light dark matter region, $m_{S} \lesssim 1 \mathrm{GeV}$, is ruled out by a large branching ratio of the $B \rightarrow K S S$ decay [25, 26, 27, 28. It has been studied that a heavier region can be explored by the invisible decay of $\Upsilon$ (or other heavy meson) [29]. Inside the dashed line, the model is perturbative and the Higgs potential is stable up to the Planck scale. It is interesting to notice that the recent experimental results started to explore a parameter region of the model.

We also show contours of $B_{\text {inv }}$ (solid lines) on the same plane. In the dark matter detection and the Higgs search experiments, there are in principle four physical observables, $m_{h}, B_{\text {inv }}, m_{S}$, and $\sigma_{S I}$, whereas in this model $B_{\text {inv }}$ and $\sigma_{S I}$ can be calculated in terms of other two, $m_{h}$ and $m_{S}$. Therefore, one can check the consistency by using data from both experiments. Almost entire region in Fig.1 will be covered by next generation experiments such as SuperCDMS, XENON, and XMASS [21, 30, 31] except for a region very close to the $m_{S}=m_{h} / 2$ line. Also, the Higgs boson search at the LHC will cover most of the region either by the invisible mode or by the ordinary standard model Higgs searches.

Finally, we comment on the case where two events reported by CDMS II is a signal of dark matter. In this case, the parameter region is restricted near the boundary of the CDMS II exclusion region. Interestingly, the mass region preferred by the data, $m_{S} \lesssim 100 \mathrm{GeV}$ [32, 11], is consistent with the prediction in Fig. 11. Since $B_{\text {inv }}$ is rather large in the region, the LHC experiments should confirm the scenario by observing the invisible decay of the Higgs boson when $m_{h} \lesssim 160 \mathrm{GeV}$.

The positron excess reported by the PAMELA experiment [33] cannot be simultaneously explained. One can assume that decays of dark matter provide a source of high-energy positrons through a small breaking of the $Z_{2}$ symmetry. In order to explain the spectrum, $m_{S}$ is required to be larger than $200 \mathrm{GeV}$, that is not compatible with

\footnotetext{
${ }_{\ddagger}^{\ddagger}$ It may be possible to discover the invisible mode even for $m_{h} \gtrsim 160 \mathrm{GeV}$, i.e., $\xi^{2} \sim 30 \%$, once systematic uncertainties are better understood [19].
} 
the explanation of the CDMS II data in this model.

\section{Acknowledgments}

This work was supported in part by the Grant-in-Aid for the Global COE Program Weaving Science Web beyond Particle-matter Hierarchy from the Ministry of Education, Culture, Sports, Science and Technology of Japan (M. A.) and the Grant-in-Aid for Scientific Research from the Ministry of Education, Science, Sports, and Culture of Japan, no. 21840006 (R.K.).

\section{References}

[1] V. Silveira and A. Zee, Phys. Lett. B 161, 136 (1985).

[2] J. McDonald, Phys. Rev. D 50, 3637 (1994) [arXiv:hep-ph/0702143].

[3] C. P. Burgess, M. Pospelov and T. ter Veldhuis, Nucl. Phys. B 619, 709 (2001) [arXiv:hep-ph/0011335].

[4] J. McDonald, Phys. Rev. Lett. 88 (2002) 091304 [arXiv:hep-ph/0106249].

[5] H. Davoudiasl, R. Kitano, T. Li and H. Murayama, Phys. Lett. B 609, 117 (2005) [arXiv:hep-ph/0405097].

[6] G. Cynolter, E. Lendvai and G. Pocsik, Acta Phys. Polon. B 36 (2005) 827 [arXiv:hep-ph/0410102].

[7] V. Barger, P. Langacker, M. McCaskey, M. J. Ramsey-Musolf and G. Shaughnessy, Phys. Rev. D 77 (2008) 035005 [arXiv:0706.4311 [hep-ph]].

[8] X. G. He, T. Li, X. Q. Li, J. Tandean and H. C. Tsai, Phys. Rev. D 79 (2009) 023521 [arXiv:0811.0658 [hep-ph]].

[9] M. Gonderinger, Y. Li, H. Patel and M. J. Ramsey-Musolf, arXiv:0910.3167 [hep$\mathrm{ph}]$.

[10] X. G. He, T. Li, X. Q. Li, J. Tandean and H. C. Tsai, arXiv:0912.4722 [hep-ph].

[11] M. Farina, D. Pappadopulo and A. Strumia, arXiv:0912.5038 [hep-ph].

[12] Z. Ahmed et al. [The CDMS Collaboration], arXiv:0912.3592 [astro-ph.CO].

[13] The CDF Collaboration et al. arXiv:0911.3930 [hep-ex]. 
[14] G. Hinshaw et al. [WMAP Collaboration], Astrophys. J. Suppl. 180 (2009) 225.

[15] A. Corsetti and P. Nath, Phys. Rev. D 64 (2001) 125010 [arXiv:hep-ph/0003186].

[16] H. Ohki et al., Phys. Rev. D 78 (2008) 054502 [arXiv:0806.4744 [hep-lat]].

[17] H. Y. Cheng, Phys. Lett. B 219 (1989) 347.

[18] H. Ohki et al., arXiv:0910.3271 [hep-lat].

[19] G. Aad et al. [The ATLAS Collaboration], arXiv:0901.0512 [hep-ex].

[20] A. Djouadi, J. Kalinowski and M. Spira, Comput. Phys. Commun. 108 (1998) 56 [arXiv:hep-ph/9704448].

[21] E. Aprile, L. Baudis and f. t. X. Collaboration, arXiv:0902.4253 [astro-ph.IM].

[22] R. Barate et al. [LEP Working Group for Higgs boson searches et al.], Phys. Lett. B 565 (2003) 61 [arXiv:hep-ex/0306033].

[23] [LEP Higgs Working for Higgs boson searches et al.], arXiv:hep-ex/0107032.

[24] ALEPH Collaboration et al., arXiv:0911.2604 [hep-ex].

[25] M. C. Bento, O. Bertolami and R. Rosenfeld, Phys. Lett. B 518 (2001) 276 [arXiv:hep-ph/0103340].

[26] C. Bird, P. Jackson, R. V. Kowalewski and M. Pospelov, Phys. Rev. Lett. 93 (2004) 201803 [arXiv:hep-ph/0401195].

[27] C. Bird, R. V. Kowalewski and M. Pospelov, Mod. Phys. Lett. A 21 (2006) 457 [arXiv:hep-ph/0601090].

[28] C. S. Kim, S. C. Park, K. Wang and G. Zhu, arXiv:0910.4291 [hep-ph].

[29] G. K. Yeghiyan, arXiv:0909.4919 [hep-ph].

[30] K. Abe [XMASS Collaboration], J. Phys. Conf. Ser. 120 (2008) 042022.

[31] P. L. Brink et al. [CDMS-II Collaboration], In the Proceedings of 22nd Texas Symposium on Relativistic Astrophysics at Stanford University, Stanford, California, 13-17 Dec 2004, pp 2529 [arXiv:astro-ph/0503583].

[32] J. Kopp, T. Schwetz and J. Zupan, arXiv:0912.4264 [hep-ph].

[33] O. Adriani et al. [PAMELA Collaboration], Nature 458 (2009) 607 [arXiv:0810.4995 [astro-ph]]. 